\title{
Study on the Reform and Innovation of Juvenile Sports Training Mode in China
}

\author{
Wei Liu \\ Hunan Vocational College of Modern Logistics, Changsha, Hunan, 410131
}

Keywords: juvenile sports; training mode; reform and innovation

\begin{abstract}
There are many unreasonable factors in the juvenile sports training and competition organization model in China. Therefore, it is necessary to change the model of government-led and vertical linkage in the past, and replace it with a model that aims to cultivate social interests, and to make horizontal connections. The key to incorporating amateur sports schools into the general school system as a transitional measure is that the sports department and the education department should be well integrated, and a sound association system should be established to clearly define the association in the form of legislation.
\end{abstract}

\section{Introduction}

After nearly 10 years of exploration, China's competitive sports have made considerable progress in the reform of management system and operational mechanism, but the problems of China's youth and children's sports training and competition system, under the long-term planned economic conditions, accumulated contradictions, Far from being fundamentally resolved, since the mid-1980s, the development of sports training for young children in China has been stagnant and the scale has shrunk. Under this background, the author studies the reform of amateur sports training and competition system for young children. Provide policy recommendations for relevant departments.

\section{Status Quo of Juvenile Sports Training}

(1) Document data method. Through the literature search method, investigators reviewed the journals and articles on youth sports training. This provides a certain theoretical basis for this investigation and research activity. (2) questionnaire survey. A total of 400 young people and 10 physical education teachers from Hailar District, Hulunbeier City, Inner Mongolia Autonomous Region were randomly selected as research objects. A total of 400 young people participated in the questionnaire survey. Among the 400 questionnaires sent out, 385 were returned, with a recovery rate of $96.25 \%$, 380 valid questionnaires, and an effective rate of 95\%. (3) Mathematical statistics. The data and data obtained from the survey were statistically processed using SPSS 13.0 software.

Students' motivation to participate in sports training. According to the data from the survey and research activities, as a sports training activity aimed at developing students' physical functions, up to $71.4 \%$ of the young people chose sports training for their physical fitness; secondly, $68.8 \%$ The youngsters participate in sports training because they like sports. This fully shows that contemporary young people have a certain degree of interest in sports. Of course, this is inseparable from the functional value of sports training itself. In addition, 21\% of young people think that physical training can shape the body, they chose sports training for the purpose of bodybuilding and weight loss. (2) Students' interest analysis. From the survey data, we can see that contemporary youth are enthusiastic about sports training. Only $8.16 \%$ of young people are not interested in sports training. They think that the training process is very laborious and can lead to physical exhaustion. From the data we can see that sports training is more popular among contemporary adolescents, and it also reflects that young people have a higher desire for participation in sports training.

According to the survey, $82.4 \%$ of the young people are not satisfied with the training content. At its root, it is mainly that some current coaches' perceptions of sports training still remain in the normal routine training. They cannot clearly understand the connotation of sports training, and most 
of them are in a state of groping and practicing. Training is limited to general training. The number of young people who chose the two options of "not satisfied" and "very dissatisfied" accounted for 73.03\% of the total number, which was more than half. Judging from the actual situation of China's current youth sports training, the situation is not optimistic. There are still problems such as a single training method and excessive training load. This has led to low effect of sports training, which has seriously affected the effectiveness of training, resulting in some young people who like physical exercise. Do not like sports training.

\section{Effective Methods for Juvenile Sports Training}

This method of sports training is a method that helps young people to perform integrity exercises. It is mainly used in the process of technical actions or tactical cooperation. In general, the complete training method can be used for a single technical movement training, but also can be used in some collective tactical training. Combined with the youth sports training practice, it can be known that when using the complete training method, the coach must emphasize the integrity of the training action, and the training links must be closely linked. Of course, for some more complex sports training programs, such as Tai Chi, Chang Quan, etc., especially the action of decomposition teaching, it is not appropriate to use this training method.

When training a single action, you should pay attention to the close connection between the actions and maintain the integrity of the action. In other words, the coaches should form the whole training link as a whole, and gradually improve the training load intensity while ensuring the improvement of the training quality.

In the training of diversified actions, it is necessary to base on its "diversity", find the connection point of the action training, and connect the various actions. In this process, we must focus on training, especially for some movements and key movements with high difficulty coefficient, we must focus on training; then we can link a whole set of movements for fusion training (complete training) . Of course, in the process of training, the coaches should carefully and subtly observe the training of the young people. Once they are found to be inadequate, they should be corrected in time, and then gradually guide them to connect a set of actions and repeat the practice. According to the actual needs of sports training programs and training actions, coaches should make a gradual transition when choosing auxiliary practice projects, and avoid making them one step at a time. For example, when training hurdles, you can use auxiliary exercises such as ligament stretching, low-column, and over-column. This not only helps young people develop physical fitness, but also helps them gradually adapt to hurdles and master the movement skills. Key points.

Decomposition training method, as the name implies, is a scientific and rational division of a complete set of training actions into several parts, and then a part-by-part method of training. This training method can reduce the degree of difficulty of training items or movements, help young people to receive training, and gradually grasp the skills of movement; at the same time, it can also highlight the difficult points of training by means of decomposition, and in the training of heavy and difficult points, Coaches can practice more. Of course, the decomposition training method also has certain deficiencies. The disadvantage is that it may lead to the formation of the habit of adolescents to break down the action. Once this habit is formed, the teenagers will divide the actions into independent ones during the practice. Blocks, it is difficult to form an overall concept in the mind, obstructing young people to master the training action completely and smoothly. Therefore, when carrying out sports training, coaches should apply this method to some complex, decomposable, and difficult to master movement training teaching.

\section{Strategies for Organizing Juvenile Sports Training and Competition Associations}

It is necessary to establish a macro, meso and micro-level structure of the government, youth sports training and competition organization associations, grass-roots schools or clubs, to clarify the positioning of the three, and to clarify the respective government sports management departments and the youth sports training and competition organization associations. Functions should regulate 
the organization and activities of the association through laws and regulations, protect its rights and interests, confirm its status, nature and functions, and use legal means to supervise and manage it. This requires the foundation of the Sports Law. On the supporting legislation.

Representative of the Association of Sports Training and Competition Organizations for Young Children. The association should develop members across regions and systems, so that its members can cover all grassroots training units in a certain geographical area.

Self-discipline of management. The Youth and Children's Sports Training and Competition Organization Association exercises the self-discipline management of the interests of all grassroots members and promotes development.

The breadth of information. The Youth and Children's Sports Training and Competition Organization Association shall timely and accurately grasp and publish the latest information on sports training and competition for young children through contact and exchange with grassroots members and domestic and foreign counterparts.

The social nature of the activities. The Youth Sports Training and Competition Organization Association has a foothold in youth sports activities, and the stage of activities is in society. The association shall establish contact with all aspects of sociality, civility, reciprocity, mediation, and social aspects. From the government to the general public, they can contact each other so as to set up primary schools or clubs and the government, as well as the society. The bridge of the aspect. Professionalism of the service. The advantage of the Youth Sports Training and Competition Organization Service is to bring together a team of experts who love, understand and understand the sports training and competition of young children. Their knowledge and skills enable all grassroots units to receive good service.

Specifically, it is necessary to cultivate five advantages. (1) Representatives of youth sports training and competition organization associations. The association must develop members across regions and systems to enable its members to cover all grassroots training units within a certain geographical area. (2) Self-discipline of management. The Youth Sports Training and Competition Organization Association exercised the safeguarding of the interests of all grass-roots members and promoted self-disciplined management of development. (3) Extensive information. The Youth Sports Training and Competition Organization Association shall timely and accurately grasp and publish the latest information on youth sports training and competitions through contacts and exchanges with grassroots members and domestic and foreign counterparts. (4) To carry out social activities. The Youth Sports Training and Competition Organization Association has a foothold in youth sports activities and carries out activities on the stage in society. Associations must establish links with all aspects of society through social, non-governmental, reciprocal, and intermediaries, from the government to the ordinary people, so that they can establish grassroots schools or clubs and governments, and all aspects of society. Bridge. (5) Professionalism of service. The advantage of the Youth Sports Training and Competition Organization Association service is to bring together a team of experts who love, understand and understand the youth sports training and competition business. Their knowledge and skills can make the grassroots units get good service.

Establishing youth sports training and competition associations is an effective means of integrating social forces. In the UK, the training of young athletes in football clubs is carried out under the unified planning of the British Football Association, the British Players Federation and the British League Committee. Through these associations, the UK Department of Labor provides funding for youth training and competitions for grassroots clubs. The Association strictly controls the training standards for young athletes and stipulates that student athletes must meet certain learning standards before they can continue to participate in training and competition. The experience of foreign countries and the practice of China for many years show that there is no association organization that links various grassroots training organizations. Each training unit is a loose sand, the operation is not standardized, the standard is not standard, the quality is not high, it is difficult to last, not to mention the scale. . 


\section{Conclusion}

Under the background of the general public's advocacy of sunshine sports and fitness, it is inevitable to strengthen the physical training of contemporary youth. Under the new situation, coaches should base on the current situation of youth sports training, and look at the misunderstandings and bottlenecks in training, review and reflect on the original training, better carry out training activities, highlight the charm of sports, and realize sports careers. Continue to develop.

\section{References}

[1] Dou Lintao. Exploration of Youth Sports Training Method [J]. Eiko: Upper and middle, 2015 (16).

[2] Xie Shengying, Sun Ying. Research on strengthening ideological and moral education in youth sports training [J]. Juvenile Sports Training, 2008 (1).

[3] Ruan Yaping. On the training methods for small and medium venues in sports training [J]. Science, Education, and Culture: Late Journal, 2010(4).

[4] Wu Yugang. On the reform of amateur training in schools [J]. Journal of Shanghai Physical Education Institute, 1999, (2): 5-8.

[5] Lu Yuanzhen. Retrospect and Prospect of Sports Development at the Turn of the Century [J]. Sports Science, 2000, (3): 1-3. 